\title{
Phenotypic diversity of date palm cultivars (Phoenix dactylifera L.) from Algeria
}

\section{Diversidad fenotípica de cultivares de palmera datilera (Phoenix dactylifera L.) de Argelia}

\author{
Ahmed Simozrag ${ }^{1 *}$, Adel Chala ${ }^{2}$, Aissa Djerouni ${ }^{1}$ \& Mouhamad Elmoncef Bentchikou ${ }^{1}$ \\ ${ }^{1}$ Laboratoire de Développement et Valorisation des Ressources Phytogénétiques. Université des Frères Mentouri Constantine, \\ Algérie. \\ ${ }^{2}$ Laboratoire de Mathématiques Appliquees. Université Mouhamed Khider. P.O. Box 145, Biskra 07000, Algérie. \\ *simozragahmed@yahoo.fr; a.simozrag@univ-biskra.dz.
}

\begin{abstract}
The aim of this work is to evaluate the phenotypic diversity of date palm (Phoenix dactylifera L.). This study shows that Biskra region has an interesting biodiversity. This latest was conducted during the growing palm groves since 3 years $(2010,2011$ and 2012) in Biskra, on 89 date palm varieties, to identify their distinctive phenotypic characteristics. Seventeen physical parameters have been examined, including 4 relative to vegetative growth, and 13 to the fruits at the date parameters stage. The main components analysis was used to identify the differences and similarities between the parameters studied. The principal component analysis showed that morphological characters for fruits should be used for identifications and descriptions of date palm cultivars. The results revealed a wide variation for different degrees of significance between the 89 cultivars for most studied traits, and showed the superiority of the fruiting characteristics over the vegetative, the former included: Fruit weight 0.851 and length 0.746 , flesh weight 0.865 , the coefficient of fruit length/width 0.754 and the weight coefficient flesh/seed 0.675 . These features had significant representation, as they helped significantly in finding 18 well identified varieties. The results analysis showed three homogeneous groups varieties sharing some traits, the convergence rate is different from one class to another within the same group. This study allowed us to highlight the most discriminating characters that can be used for further studies of diversity.
\end{abstract}

KEYwORDs: Cultivars, date palm, identification, Algeria, phenotypic.

\section{RESUMEN}

El objetivo de este trabajo es evaluar la diversidad fenotípica de la palmera datilera (Phoenix dactylifera L.). Nuestro estudio muestra que la región de Biskra cuenta con una interesante biodiversidad. Este estudio se llevó a cabo durante 3 años de crecimiento $(2010,2011$ y 2012) en Biskra, para 89 variedades de palmera datilera, para identificar sus características fenotípicas distintivas. Se analizaron 17 parámetros físicos, 4 de ellos relacionados al crecimiento vegetativo y 13 durante la etapa de fruto. Se utilizó el análisis de componentes principales para identificar las diferencias y similitudes entre los parámetros estudiados. El análisis de componentes principales mostró que los caracteres morfológicos de las frutas deben ser utilizados para identificaciones y descripciones de variedades de palmera datilera. Los resultados revelaron una amplia variación para diferentes grados de importancia entre los 89 cultivares para los rasgos más estudiados, y mostraron la superioridad de las características de fructificación frente a los de vegetación para distinguir las variedades. Estos resultados incluían: peso del fruto 0,851 , longitud del fruto 0,746 , peso de la pulpa del fruto 0,865 , coeficiente longitud/ancho del fruto 0,754 y coeficiente peso pulpa/semilla 0,675 . tienen representación significativa para diferenciar 18 variedades. Los resultados del análisis estadístico mostraron que hay tres grupos homogéneos de variedades que presentan similitudes en algunas de las características estudiadas, y la tasa de convergencia era diferente de una clase a otra dentro del mismo grupo. Este estudio nos ha permitido poner de relieve los caracteres discriminantes que pueden ser utilizados para otros estudios de diversidad.

Palabras clave: Cultivares, palmera datilera, identificación, Argelia, fenotípica. 


\section{INTRODUCTION}

The date palm (Phoenix dactylifera L.) is the most important fruit tree grown in Algeria and the Arab world due to its commercial and economic usefulness, where Algeria is among the leading countries in the cultivation and production of palms dates. Recent statistics indicate that the number of palm trees planted globally grown accounts for about 18 millions (Anonyme 2011).

Date palms confined in Algerian oases exceed 900 cultivars, including soft, dry and semi-dry varieties. The greater part of them is classified within the bad range in terms of quality, which is often not economically rentable and affects their plentiful and spread. Each region has its own cultivars. Some of them have been transplanted to other areas with the same or new names. Cultivars having more than one name. Or common names calling for two or more cultivars (Barrett 1975).

In date palms, most of the female cultivars are recognized by their fruit characteristics such as size, shape, colour and taste. Morphologic characters of the tree are also taken into consideration for cultivar identification. For the male trees, cultivar identification is a cumbersome process because they mostly are seed-borne and are hardly identical to any female cultivar. However, farmers dealing with date palms can identify some male cultivars from their experience. Several workers attempted to describe and enlist the date palm cultivare grown in their countryof interest. Rhouma (1994) enlisted 250 cultivars of date palm grown in Tunisia, Dowson (1923) indentified 370 cultivars in Iraq, while 26 cultivars were recorded by Brown (1924) from Egypt, and Nixon (1950) enlisted 196 date verieties imported in the USA. In Morocco, Saaidi (1979) identified 244 cultivars; in 1996, Bashah provided fruit description for 30 cultivars grown in Saudi Arabia. Zaid \& Wet (1999) provided an elaborative description of two cultivars, viz Mejdool and Barhee covering vegetative and reproductive characters, which can be used as amodel for detailed description of other cultivars. In Algeria, Hannachi et al. (1998) presented a study on 160 cultivars and Belguedj (2002) on 120 cultivars as well as Belguedj \& Tirichine (2011) on 75 cultivars included the phenotypic characteristics of the leaves and fruit.

Date palm varieties are very similar; however, studies have shown that there are clear differences based on the vegetative characteristics and Spath (Djerouni et al. 2015). Since the palm leave constituents look very different, the measurements which were taken from leaf palm like thorns length, pinna number and leaf palm length have shown the similarities and the differences between the palms (Saker et al. 2010, Hider et al. 2015). The measurements taken from the fruits can be considered as the best proof of the differences of varieties of date palm (Mohamed et al. 2014). Thus, to differenciate between the trees and plants that belong to the same family through vegetative characteristics.

Since the lack of studies on the phenotypic characterization of different cultivars in Algeria, and publication of the references values of the International Plant Genetic Research Institute (IPGRI), determining the criterions that can be relied upon to distinguish palms and identify the differences and similarities between cultivars, a study on date cultivars cultivated in Biskra (Algeria) have conducted to identify the distinctive phenotypic characteristics and determine the existing extent of variation.

The first objective is to contribute to the morphological characterization of 89 cultivars date palm and enrich the existing germplasm in Biskra. The study is based on a detailed morphological characterization of the vegetative part and the fruit of 89 cultivars. The second objective is the exploitation of the statistical analysis results in order to study the phenotypic diversity among cultivars in the Biskra region. This is done for the assessment of the polymorphism degree between studied and the importance of the most discriminating characters in order to have a classification of homogeneous groups and cultivars which can be used for the classification of date palm.

\section{MATERIAL AND METHODS}

The 89 varieties are shown Appendix A of the date palm that have been selected were cultivated in the oases of the Biskra region, in the south-eastern Algeria (see Fig. 1), that lies at an altitude of $124 \mathrm{~m}$ above sea level, between latitudes $35^{\circ} 15^{\prime}-33^{\circ} 30^{\prime}$ north and longitudes $04^{\circ} 15^{\prime}-06^{\circ} 45^{\prime}$, They have been estimated to cover $21,617 \mathrm{~km}^{2}$ (Anonyme 2003 ).

The vegetative activity of date palm largely linked with the climatic condition of region. The average of maximum and minimum temperature respectively $28.43{ }^{\circ} \mathrm{C}$ and 17.01 ${ }^{\circ} \mathrm{C}$ witch appropriate for the optimal species needs that vary between $32^{\circ} \mathrm{C}$ and $38^{\circ} \mathrm{C}$ and the tolerance limits that range between $7-45{ }^{\circ} \mathrm{C}$ (Khadhi et al. 1995). The Saharan rains are ineffective for irrigation $(10,96 \mathrm{~mm})$. However, they can affect flowering and maturing where abundant rainfall in spring and autumn can launch the development of diseases and parasite attacks (Belguedj et al. 2008). The ecological optimum of relatively average humidity $(\mathrm{H})$ is $40 \%$ (Belguedj et al. 2008), the latter is almost adequate with the value of humidity in the study area $(42.2 \%)$, allowing them to keep textures in relation to their varietal characteristics (Belguedj et al. 2008). Finally, the wind are frequent in the Spring but generally calm $(4.44 \mathrm{~m} / \mathrm{s})$ (Table I).

Three repetitions of each cultivar have been chosen as identical in force of their growth, the similar size of each class of age (15 years), and because all the palm trees have been treated as the practice with each other in terms of irrigation, pruning, pollination and other agricultural operations. 
Quantitative parameters for each variety have been also studied, including (Table II): 4 parameters for leaves and 13 for fruits (natural qualities), where 3 leaves and 20 fruits in the phase of dates were taken from each varieties (Anonyme 2005). The Figure (2) shows the description of some essential parameters of leaf and fruit of date palm.
DATA ANALYSES

Principal Component Analysis (PCA) was used (Johnston 1978, Mainley 1994). In order to identify groups of intercorrelated variables for $P$. dactylifera. Data analyses were performed using XLSTAT program (2009.3.02) which allowed us to make the intra-cultivar variance analysis. The stable selected descriptor measurements of the cultivars were subjected to a components analysis to determine the characteristics with greater contributions to the total variability.
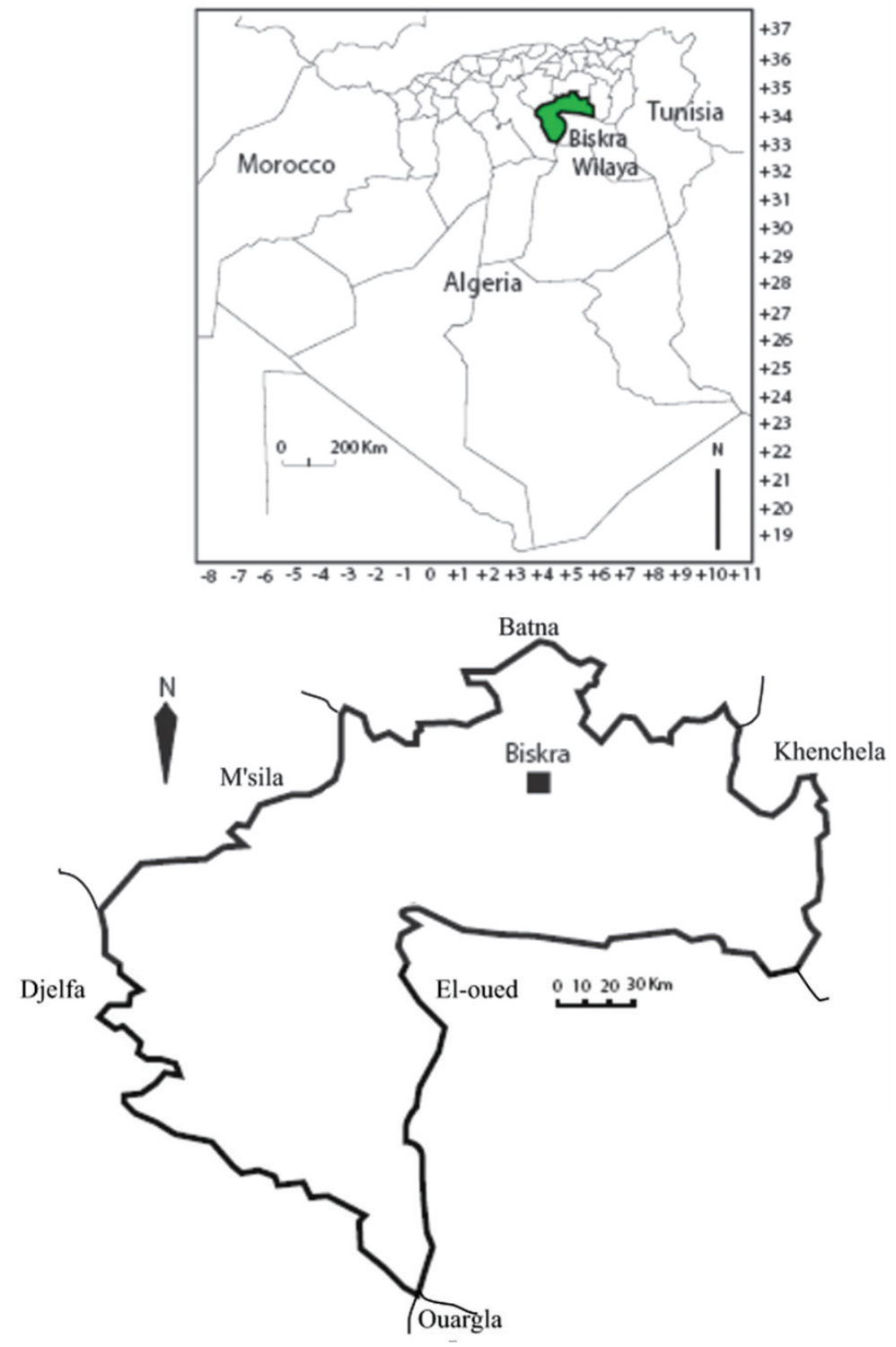

Figure 1. Map of Biskra.

Figura 1. Mapa de Biskra. 
Phenotypic diversity of Phoenix dactylifera: Simozrag, A. ET AL.

TABLE I. Climate data from the Biskra region for the period 2002-2012.

TABLA I. Los datos climáticos de la región de Biskra para el período 2002-2012.

\begin{tabular}{|c|c|c|c|c|c|c|c|c|c|}
\hline \multirow{2}{*}{ MonTH } & \multicolumn{2}{|c|}{$\mathrm{T}\left({ }^{\circ} \mathrm{C}\right)$} & \multicolumn{2}{|c|}{$\operatorname{Tm}\left({ }^{\circ} \mathrm{C}\right)$} & \multicolumn{2}{|c|}{$\mathrm{TM}\left({ }^{\circ} \mathrm{C}\right)$} & \multirow{2}{*}{$\mathrm{P}(\mathrm{mm})$} & \multirow{2}{*}{$\mathrm{V}(\mathrm{m} / \mathrm{s})$} & \multirow{2}{*}{$\mathrm{H}(\%)$} \\
\hline & moy & e-type & moy & e-type & moy & e-type & & & \\
\hline $\mathrm{J}$ & 6.27 & 11.25 & 1.08 & 1.24 & 16.91 & 1.36 & 26.42 & 4.18 & 59.00 \\
\hline $\mathrm{F}$ & 7.82 & 13.12 & 1.41 & 1.17 & 19.11 & 1.95 & 6.02 & 4.4 & 48.64 \\
\hline M & 11.76 & 17.61 & 1.36 & 1.04 & 23.87 & 1.50 & 12.20 & 5.05 & 41.45 \\
\hline A & 15.39 & 21.25 & 1.28 & 0.74 & 27.52 & 1.27 & 11.85 & 5.88 & 37.45 \\
\hline M & 20.59 & 26.90 & 1.60 & 1.38 & 31.99 & 2.82 & 11.45 & 5.61 & 32.73 \\
\hline $\mathrm{J}$ & 25.00 & 31.93 & 1.17 & 0.96 & 38.11 & 1.23 & 1.39 & 4.37 & 27.27 \\
\hline $\mathrm{J}$ & 28.01 & 34.89 & 1.08 & 1.12 & 41.20 & 1.13 & 0.85 & 4.00 & 25.82 \\
\hline A & 27.78 & 34.22 & 0.82 & 0.97 & 39.80 & 1.30 & 2.47 & 3.76 & 28.36 \\
\hline $\mathrm{S}$ & 23.19 & 28.78 & 0.93 & 0.91 & 34.01 & 1.47 & 15.90 & 4.07 & 41.18 \\
\hline $\mathrm{O}$ & 18.66 & 23.94 & 1.29 & 1.20 & 29.65 & 1.69 & 12.00 & 3.65 & 47.45 \\
\hline $\mathrm{N}$ & 11.78 & 16.02 & 1.76 & 0.79 & 21.79 & 0.79 & 15.07 & 4.15 & 54.27 \\
\hline $\mathrm{D}$ & 7.90 & 12.17 & 1.00 & 0.94 & 17.21 & 1.21 & 15.85 & 4.20 & 62.73 \\
\hline Avg/year & 17.01 & 22.67 & 1.23 & 1.04 & 28.43 & 1.48 & 10.96 & 4.44 & 42.2 \\
\hline
\end{tabular}

T: Average Temperature $\left({ }^{\circ} \mathrm{C}\right)$; Tm: Minimum Temperature $\left({ }^{\circ} \mathrm{C}\right)$; TM: Maximum temperature $\left({ }^{\circ} \mathrm{C}\right)$; P: Total rainfall $(\mathrm{mm})$; V: Average wind speed $(\mathrm{m} / \mathrm{s})$; H: Average relative humidity $(\%)$. / T: Temperatura media $\left({ }^{\circ} \mathrm{C}\right)$; Tm: Temperatura mínima $\left({ }^{\circ} \mathrm{C}\right)$; TM: Temperatura máxima $\left({ }^{\circ} \mathrm{C}\right)$; P: Precipitación total de lluvia $(\mathrm{mm}) ; \mathrm{V}$ : Velocidad media del viento $(\mathrm{m} / \mathrm{s}) ; \mathrm{H}$ : Humedad relativa media $(\%)$.
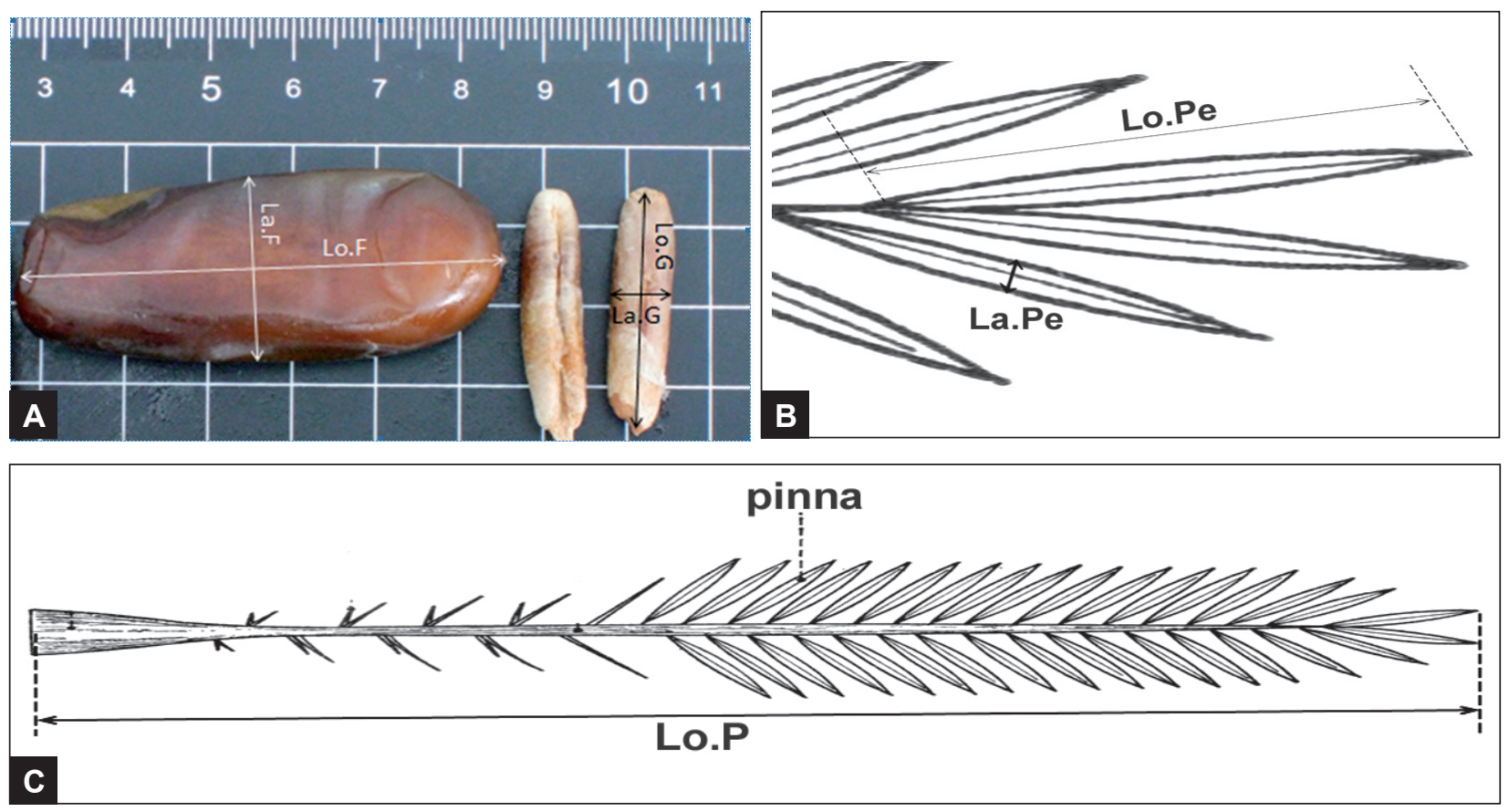

Figure 2. A. The fruit and seeds. B. The pinna. C. The leaf. Descriptors in Table II.

Figura 2. A. El fruto y semillas. B. La pinna. C. La hoja. Descriptores en Tabla II. 
TABLA II. Los valores medios de los caracteres fenotípicos de cultivares datileras.

\begin{tabular}{|c|c|c|c|c|c|c|c|c|c|c|c|c|c|c|c|c|c|}
\hline 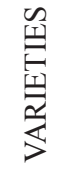 & 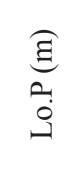 & $\begin{array}{l}0 \\
0 \\
0 \\
z\end{array}$ & 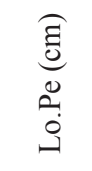 & 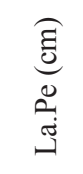 & $\underset{\Xi}{\stackrel{a}{\Xi}}$ & $\frac{e^{0}}{\sum^{n}}$ & $\underbrace{000}_{0.1}$ & હ્ఏ్ఏ & 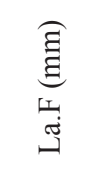 & & $\begin{array}{l}\text { क्रि } \\
0 \\
0 .\end{array}$ & 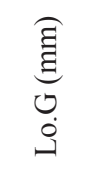 & 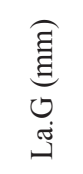 & 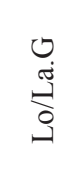 & : & نب & 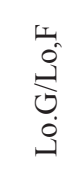 \\
\hline $\mathrm{V} 1$ & 2.64 & 116.44 & 50.00 & 3.77 & 20.93 & 79.07 & 12.04 & 37.41 & 22.38 & 1.67 & 1.39 & 23.97 & 8.15 & 2.94 & 10.66 & 7.67 & 0.64 \\
\hline $\mathrm{V} 2$ & 2.75 & 139.00 & 48.89 & 2.61 & 17.27 & 82.73 & 5.92 & 28.27 & 18.68 & 1.51 & 0.71 & 22.68 & 6.93 & 3.27 & 5.22 & 7.39 & 0.80 \\
\hline V3 & 3.37 & 130.89 & 35.44 & 2.29 & 14.20 & 85.80 & 7.57 & 35.88 & 18.15 & 1.98 & 0.68 & 17.60 & 7.08 & 2.49 & 6.90 & 10.19 & 0.49 \\
\hline V4 & 2.73 & 147.33 & 47.00 & 2.72 & 16.27 & 83.73 & 5.50 & 33.97 & 15.84 & 2.15 & 0.98 & 22.95 & 7.24 & 3.17 & 4.51 & 4.60 & 0.68 \\
\hline V5 & 5.16 & 192.44 & 53.33 & 4.44 & 14.40 & 85.60 & 4.17 & 30.36 & 16.71 & 1.82 & 0.96 & 19.98 & 8.11 & 2.46 & 3.21 & 3.36 & 0.66 \\
\hline V6 & 4.08 & 208.44 & 53.78 & 2.86 & 22.37 & 77.63 & 7.80 & 35.53 & 17.64 & 2.01 & 0.67 & 23.73 & 7.48 & 3.17 & 7.13 & 10.60 & 0.67 \\
\hline V7 & 2.99 & 115.78 & 46.89 & 3.77 & 20.47 & 79.53 & 13.73 & 41.93 & 22.54 & 1.86 & 1.23 & 24.96 & 8.04 & 3.10 & 12.50 & 10.13 & 0.60 \\
\hline V8 & 4.19 & 271.33 & 46.11 & 4.06 & 20.90 & 79.10 & 16.19 & 49.22 & 18.03 & 2.73 & 0.77 & 18.75 & 7.34 & 2.55 & 15.42 & 20.02 & 0.38 \\
\hline V9 & 3.67 & 173.67 & 42.11 & 3.67 & 15.27 & 84.73 & 5.01 & 32.69 & 26.01 & 1.26 & 1.00 & 22.90 & 7.34 & 3.12 & 4.01 & 4.01 & 0.70 \\
\hline V10 & 2.90 & 140.33 & 44.89 & 2.42 & 22.47 & 77.53 & 6.24 & 37.76 & 19.13 & 1.97 & 1.07 & 22.98 & 7.66 & 3.00 & 5.17 & 4.84 & 0.61 \\
\hline V11 & 3.75 & 199.89 & 55.22 & 3.24 & 25.00 & 75.00 & 9.43 & 36.81 & 23.47 & 1.57 & 0.83 & 22.82 & 6.55 & 3.48 & 8.60 & 10.43 & 0.62 \\
\hline V12 & 4.06 & 176.89 & 52.67 & 3.66 & 32.20 & 67.80 & 12.84 & 39.61 & 17.03 & 2.33 & 1.20 & 24.89 & 7.75 & 3.21 & 11.63 & 9.66 & 0.63 \\
\hline V13 & 3.89 & 200.89 & 60.33 & 3.28 & 11.50 & 88.70 & 5.81 & 38.45 & 17.17 & 2.24 & 1.10 & 25.11 & 6.21 & 4.04 & 4.71 & 4.28 & 0.65 \\
\hline V14 & 3.72 & 193.67 & 41.89 & 3.43 & 12.05 & 87.95 & 8.41 & 38.20 & 20.09 & 1.90 & 1.01 & 24.56 & 7.27 & 3.38 & 7.40 & 7.30 & 0.64 \\
\hline V15 & 2.13 & 123.00 & 44.56 & 3.36 & 15.97 & 84.03 & 7.39 & 34.63 & 20.70 & 1.67 & 0.91 & 22.87 & 7.61 & 3.01 & 6.48 & 7.14 & 0.66 \\
\hline V16 & 3.64 & 174.00 & 58.22 & 3.64 & 37.31 & 62.69 & 15.22 & 48.22 & 16.25 & 2.97 & 1.08 & 24.88 & 6.93 & 3.59 & 14.14 & 13.07 & 0.52 \\
\hline V17 & 3.36 & 173.44 & 69.11 & 2.69 & 17.03 & 82.97 & 6.45 & 36.54 & 17.17 & 2.13 & 0.95 & 23.47 & 7.43 & 3.16 & 5.50 & 5.79 & 0.64 \\
\hline V18 & 4.12 & 217.67 & 53.67 & 2.97 & 14.67 & 85.33 & 5.40 & 29.87 & 20.89 & 1.43 & 1.16 & 23.27 & 6.84 & 3.40 & 4.24 & 3.64 & 0.78 \\
\hline V19 & 2.58 & 137.00 & 41.89 & 2.22 & 16.87 & 83.13 & 7.44 & 34.22 & 19.11 & 1.79 & 1.32 & 22.61 & 8.51 & 2.66 & 6.12 & 4.62 & 0.66 \\
\hline V20 & 2.54 & 130.00 & 38.89 & 3.08 & 25.53 & 74.47 & 9.49 & 44.16 & 14.83 & 2.98 & 0.85 & 24.41 & 5.84 & 4.18 & 8.65 & 10.20 & 0.55 \\
\hline V21 & 4.31 & 134.22 & 40.89 & 2.57 & 9.90 & 90.10 & 5.24 & 33.87 & 15.34 & 2.21 & 0.99 & 22.50 & 6.93 & 3.25 & 4.26 & 4.31 & 0.66 \\
\hline V22 & 3.63 & 185.89 & 46.00 & 2.30 & 14.90 & 85.10 & 3.82 & 27.72 & 15.64 & 1.77 & 0.91 & 20.19 & 7.90 & 2.55 & 2.91 & 3.21 & 0.73 \\
\hline V23 & 3.60 & 136.11 & 46.56 & 2.84 & 25.07 & 74.60 & 5.74 & 30.05 & 19.23 & 1.56 & 0.85 & 21.96 & 8.01 & 2.74 & 4.89 & 5.77 & 0.73 \\
\hline V24 & 3.59 & 216.67 & 56.11 & 2.83 & 10.10 & 89.90 & 7.40 & 58.76 & 17.92 & 3.28 & 1.38 & 28.75 & 8.48 & 3.39 & 6.02 & 4.36 & 0.49 \\
\hline V25 & 3.93 & 178.56 & 60.44 & 3.28 & 15.20 & 84.80 & 3.88 & 31.71 & 16.67 & 1.90 & 1.12 & 22.55 & 7.65 & 2.95 & 2.76 & 2.47 & 0.71 \\
\hline V26 & 3.24 & 123.67 & 48.67 & 3.89 & 19.77 & 80.23 & 9.21 & 41.86 & 18.81 & 2.22 & 0.94 & 25.82 & 7.08 & 3.64 & 8.26 & 8.77 & 0.62 \\
\hline V27 & 3.02 & 125.11 & 55.33 & 3.40 & 20.30 & 79.70 & 7.97 & 32.79 & 18.76 & 1.75 & 0.92 & 21.21 & 7.88 & 2.69 & 7.06 & 7.71 & 0.65 \\
\hline V28 & 3.00 & 115.78 & 56.00 & 3.20 & 20.23 & 79.77 & 12.91 & 41.77 & 19.81 & 2.11 & 0.92 & 24.24 & 7.61 & 3.19 & 11.99 & 12.99 & 0.58 \\
\hline V29 & 2.25 & 157.33 & 32.33 & 3.71 & 19.33 & 80.67 & 6.74 & 31.22 & 19.23 & 1.62 & 1.09 & 21.30 & 8.13 & 2.62 & 5.64 & 5.16 & 0.68 \\
\hline V30 & 3.69 & 125.78 & 41.67 & 4.17 & 19.47 & 80.53 & 9.89 & 36.60 & 18.03 & 2.03 & 1.03 & 21.64 & 8.16 & 2.65 & 8.86 & 8.58 & 0.59 \\
\hline V31 & 4.10 & 190.11 & 43.78 & 3.44 & 18.64 & 81.36 & 5.17 & 43.44 & 16.68 & 2.61 & 1.01 & 26.59 & 8.52 & 3.12 & 4.16 & 4.11 & 0.61 \\
\hline V32 & 3.47 & 184.44 & 60.78 & 2.66 & 11.27 & 91.73 & 6.18 & 34.46 & 17.10 & 2.02 & 0.79 & 22.33 & 6.96 & 3.21 & 5.39 & 6.82 & 0.65 \\
\hline
\end{tabular}




\begin{tabular}{|c|c|c|c|c|c|c|c|c|c|c|c|c|c|c|c|c|c|}
\hline 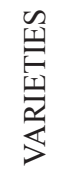 & 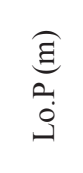 & $\begin{array}{l}0 \\
0 \\
0 \\
z\end{array}$ & 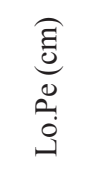 & 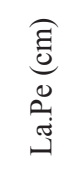 & 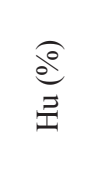 & $\frac{e^{\infty}}{\sum^{\infty}}$ & $\begin{array}{l}\text { త્00 } \\
\text { [1: }\end{array}$ & 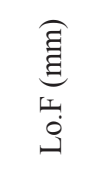 & 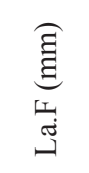 & & $\begin{array}{l}\text { (0) } \\
0 \\
0\end{array}$ & 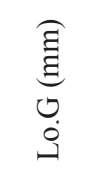 & 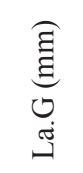 & 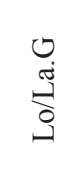 & $\underset{20}{\stackrel{0}{0}}$ & 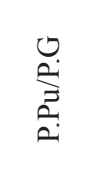 & 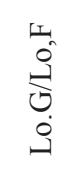 \\
\hline V33 & 4.04 & 170.00 & 46.33 & 3.43 & 21.17 & 78.83 & 7.42 & 34.94 & 19.65 & 1.78 & 1.13 & 21.74 & 8.36 & 2.60 & 6.29 & 5.56 & 0.62 \\
\hline V34 & 3.63 & 87.11 & 52.44 & 3.60 & 16.10 & 83.90 & 10.72 & 39.43 & 20.53 & 1.92 & 1.15 & 21.99 & 9.05 & 2.43 & 9.57 & 8.32 & 0.56 \\
\hline V35 & 3.20 & 157.00 & 45.56 & 3.36 & 39.37 & 60.63 & 11.82 & 38.95 & 24.13 & 1.61 & 0.82 & 21.05 & 6.63 & 3.18 & 11.00 & 13.45 & 0.54 \\
\hline V36 & 3.03 & 175.00 & 39.11 & 2.79 & 16.73 & 83.27 & 8.62 & 39.56 & 18.64 & 2.12 & 1.05 & 24.78 & 7.93 & 3.13 & 7.56 & 7.17 & 0.63 \\
\hline V37 & 3.03 & 173.67 & 38.33 & 2.79 & 21.17 & 78.83 & 5.66 & 29.33 & 19.06 & 1.54 & 1.23 & 20.33 & 8.05 & 2.53 & 4.43 & 3.60 & 0.69 \\
\hline V38 & 3.74 & 204.33 & 49.67 & 4.53 & 19.13 & 80.87 & 6.71 & 36.05 & 16.64 & 2.17 & 1.06 & 23.51 & 8.14 & 2.89 & 5.65 & 5.34 & 0.65 \\
\hline V39 & 3.32 & 138.00 & 50.44 & 2.44 & 18.93 & 81.07 & 6.44 & 37.78 & 16.18 & 2.34 & 1.00 & 25.29 & 7.00 & 3.61 & 5.43 & 5.43 & 0.67 \\
\hline V40 & 3.46 & 134.44 & 53.78 & 2.80 & 27.23 & 72.77 & 5.98 & 31.50 & 21.07 & 1.50 & 0.77 & 21.38 & 7.82 & 2.73 & 5.21 & 6.74 & 0.68 \\
\hline V41 & 3.22 & 105.56 & 45.33 & 2.29 & 23.03 & 76.97 & 14.62 & 35.53 & 21.49 & 1.65 & 1.16 & 22.77 & 8.24 & 2.77 & 13.46 & 11.59 & 0.64 \\
\hline V42 & 3.04 & 166.56 & 46.11 & 2.59 & 18.23 & 81.77 & 9.45 & 35.23 & 17.31 & 2.03 & 1.12 & 19.82 & 8.25 & 2.40 & 8.33 & 7.43 & 0.56 \\
\hline V43 & 3.41 & 228.89 & 52.33 & 5.19 & 21.03 & 78.97 & 10.87 & 30.09 & 20.67 & 1.46 & 1.36 & 22.81 & 8.78 & 2.60 & 9.51 & 7.00 & 0.76 \\
\hline V44 & 3.06 & 132.78 & 41.33 & 2.47 & 15.83 & 84.17 & 8.88 & 37.05 & 21.74 & 1.70 & 1.10 & 20.97 & 7.63 & 2.75 & 7.78 & 7.04 & 0.57 \\
\hline V45 & 3.70 & 166.22 & 33.33 & 3.30 & 20.47 & 79.53 & 7.35 & 34.58 & 14.13 & 2.45 & 0.74 & 21.96 & 5.37 & 4.09 & 6.62 & 9.00 & 0.64 \\
\hline V46 & 4.42 & 211.56 & 73.22 & 4.14 & 49.17 & 50.83 & 11.63 & 51.71 & 24.54 & 2.11 & 1.10 & 23.28 & 7.02 & 3.31 & 10.53 & 9.58 & 0.45 \\
\hline V47 & 2.56 & 127.33 & 33.89 & 3.63 & 21.07 & 78.93 & 8.00 & 42.20 & 20.67 & 2.04 & 0.88 & 22.93 & 7.73 & 2.97 & 7.12 & 8.09 & 0.54 \\
\hline V48 & 4.44 & 203.78 & 53.11 & 4.24 & 24.60 & 75.40 & 3.92 & 29.20 & 19.53 & 1.49 & 0.83 & 23.71 & 6.93 & 3.42 & 3.09 & 3.70 & 0.81 \\
\hline V49 & 2.39 & 163.78 & 46.89 & 2.37 & 20.47 & 79.53 & 9.05 & 34.05 & 19.68 & 1.73 & 1.24 & 22.72 & 9.13 & 2.49 & 7.81 & 6.28 & 0.67 \\
\hline V50 & 3.69 & 179.78 & 43.33 & 3.38 & 17.70 & 82.30 & 5.82 & 32.64 & 16.84 & 1.94 & 0.82 & 22.20 & 7.34 & 3.03 & 5.00 & 6.10 & 0.68 \\
\hline V51 & 4.20 & 182.33 & 48.56 & 3.14 & 20.30 & 79.70 & 9.54 & 45.85 & 17.00 & 2.70 & 1.07 & 23.89 & 7.19 & 3.32 & 8.47 & 7.90 & 0.52 \\
\hline V52 & 3.73 & 181.44 & 61.44 & 3.32 & 16.63 & 83.37 & 8.69 & 38.37 & 18.53 & 2.07 & 1.62 & 25.63 & 8.82 & 2.91 & 7.06 & 4.35 & 0.67 \\
\hline V53 & 3.59 & 199.78 & 60.00 & 4.06 & 14.43 & 85.57 & 7.20 & 38.81 & 17.51 & 2.22 & 1.01 & 23.25 & 8.01 & 2.90 & 6.19 & 6.10 & 0.60 \\
\hline V54 & 3.23 & 111.89 & 49.22 & 2.73 & 16.50 & 83.50 & 7.99 & 39.06 & 15.96 & 2.45 & 0.75 & 22.85 & 6.84 & 3.34 & 7.24 & 9.60 & 0.59 \\
\hline V55 & 2.52 & 140.89 & 35.44 & 3.86 & 27.57 & 72.43 & 13.02 & 33.96 & 21.83 & 1.56 & 1.25 & 23.96 & 7.24 & 3.31 & 11.77 & 9.44 & 0.71 \\
\hline V56 & 3.11 & 162.78 & 48.89 & 3.39 & 13.10 & 86.90 & 5.05 & 32.96 & 14.71 & 2.24 & 0.57 & 19.24 & 6.35 & 3.03 & 4.48 & 7.79 & 0.58 \\
\hline V57 & 3.08 & 115.22 & 63.11 & 2.44 & 20.57 & 79.43 & 8.56 & 37.69 & 19.08 & 1.98 & 1.32 & 22.51 & 7.47 & 3.01 & 7.22 & 5.48 & 0.60 \\
\hline V58 & 3.83 & 132.33 & 53.89 & 4.22 & 16.00 & 84.00 & 7.34 & 39.98 & 17.50 & 2.28 & 1.33 & 20.94 & 9.20 & 2.28 & 6.02 & 4.54 & 0.52 \\
\hline V59 & 4.40 & 104.78 & 53.44 & 3.39 & 20.97 & 79.03 & 4.65 & 34.64 & 15.15 & 2.29 & 1.67 & 22.77 & 7.36 & 3.09 & 2.97 & 1.78 & 0.66 \\
\hline V60 & 2.60 & 131.33 & 43.22 & 2.67 & 19.90 & 80.10 & 9.20 & 36.25 & 18.97 & 1.91 & 1.14 & 24.11 & 7.20 & 3.35 & 8.06 & 7.06 & 0.66 \\
\hline V61 & 2.53 & 119.33 & 38.67 & 2.72 & 12.87 & 87.13 & 6.67 & 36.46 & 18.66 & 1.95 & 1.15 & 22.08 & 8.39 & 2.63 & 5.52 & 4.79 & 0.61 \\
\hline V62 & 3.05 & 161.78 & 44.89 & 2.53 & 15.80 & 84.20 & 9.82 & 44.91 & 18.27 & 2.46 & 1.31 & 25.52 & 7.41 & 3.44 & 8.51 & 6.48 & 0.57 \\
\hline V63 & 2.52 & 141.89 & 42.11 & 3.18 & 29.10 & 70.90 & 11.37 & 35.37 & 15.39 & 2.30 & 1.62 & 23.16 & 8.62 & 2.69 & 9.75 & 6.01 & 0.65 \\
\hline V64 & 4.16 & 199.56 & 53.33 & 4.37 & 16.37 & 83.63 & 9.77 & 41.40 & 18.95 & 2.19 & 1.06 & 26.27 & 6.65 & 3.95 & 8.71 & 8.19 & 0.63 \\
\hline V65 & 3.43 & 169.56 & 50.89 & 3.33 & 22.50 & 77.50 & 8.35 & 40.58 & 16.99 & 2.39 & 0.84 & 24.90 & 7.16 & 3.48 & 7.51 & 8.94 & 0.61 \\
\hline V66 & 3.14 & 111.11 & 53.78 & 2.66 & 20.63 & 79.37 & 7.20 & 31.80 & 20.58 & 1.54 & 1.30 & 23.95 & 6.29 & 3.81 & 5.90 & 4.54 & 0.75 \\
\hline V67 & 4.00 & 185.33 & 52.11 & 2.53 & 10.80 & 89.20 & 5.89 & 34.52 & 16.70 & 2.07 & 1.04 & 23.64 & 7.53 & 3.14 & 4.85 & 4.66 & 0.68 \\
\hline
\end{tabular}




\begin{tabular}{|c|c|c|c|c|c|c|c|c|c|c|c|c|c|c|c|c|c|}
\hline 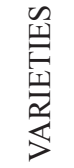 & $\begin{array}{l}\widehat{\Xi} \\
0 \\
0 \\
\hat{\jmath}\end{array}$ & $\begin{array}{l}0 \\
2 \\
\dot{2}\end{array}$ & $\begin{array}{l}\text { E्ध } \\
0 \\
0 \\
0 \\
0\end{array}$ & 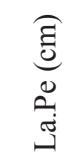 & 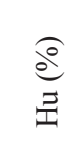 & $\frac{\varrho}{e^{0}}$ & 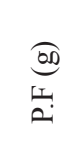 & 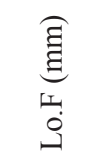 & 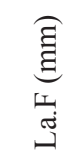 & 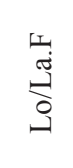 & 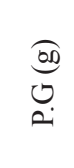 & 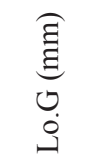 & 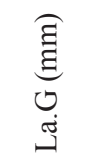 & $\begin{array}{l}0 \\
\stackrel{J}{\oplus} \\
\end{array}$ & 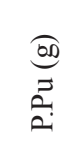 & بـ & 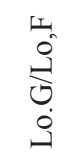 \\
\hline V68 & 2.35 & 137.00 & 44.67 & 2.78 & 19.11 & 80.89 & 7.67 & 26.35 & 18.78 & 1.40 & 1.00 & 18.55 & 10.25 & 1.81 & 6.67 & 6.68 & 0.70 \\
\hline V69 & 3.18 & 137.11 & 49.56 & 2.77 & 14.80 & 85.20 & 3.26 & 24.54 & 15.58 & 1.57 & 1.01 & 18.29 & 8.03 & 2.28 & 2.25 & 2.22 & 0.75 \\
\hline V70 & 2.55 & 101.00 & 36.22 & 2.71 & 12.97 & 87.03 & 9.87 & 42.39 & 16.83 & 2.52 & 1.51 & 26.93 & 7.42 & 3.63 & 8.36 & 5.53 & 0.64 \\
\hline V71 & 3.28 & 157.00 & 54.11 & 2.39 & 22.07 & 77.93 & 10.09 & 28.29 & 23.27 & 1.22 & 1.17 & 18.58 & 8.68 & 2.14 & 8.93 & 7.64 & 0.66 \\
\hline V72 & 3.72 & 149.78 & 50.00 & 2.96 & 17.00 & 83.00 & 4.17 & 35.59 & 23.96 & 1.49 & 1.03 & 19.75 & 8.70 & 2.27 & 3.13 & 3.03 & 0.55 \\
\hline V73 & 3.44 & 163.11 & 50.89 & 3.58 & 17.67 & 82.33 & 9.50 & 40.47 & 18.31 & 2.21 & 1.17 & 25.64 & 7.94 & 3.23 & 8.33 & 7.14 & 0.63 \\
\hline V74 & 2.95 & 162.11 & 51.11 & 2.91 & 11.23 & 88.77 & 6.03 & 32.39 & 19.58 & 1.65 & 0.80 & 20.31 & 7.48 & 2.72 & 5.23 & 6.54 & 0.63 \\
\hline V75 & 3.86 & 199.89 & 43.56 & 2.94 & 17.40 & 82.60 & 5.96 & 36.24 & 18.55 & 1.95 & 0.86 & 23.71 & 7.25 & 3.27 & 5.10 & 5.93 & 0.65 \\
\hline V76 & 4.08 & 153.44 & 53.56 & 2.34 & 16.57 & 83.43 & 6.55 & 44.48 & 16.87 & 2.64 & 0.91 & 25.88 & 7.07 & 3.66 & 5.63 & 6.16 & 0.58 \\
\hline V77 & 3.17 & 183.44 & 45.44 & 3.04 & 11.98 & 86.69 & 7.94 & 38.63 & 21.20 & 1.82 & 0.73 & 20.40 & 6.68 & 3.05 & 7.21 & 9.88 & 0.53 \\
\hline V78 & 3.86 & 215.11 & 47.11 & 2.92 & 13.20 & 86.80 & 5.71 & 33.43 & 16.02 & 2.09 & 0.84 & 21.86 & 7.25 & 3.01 & 4.88 & 5.82 & 0.65 \\
\hline V79 & 3.70 & 147.89 & 50.22 & 2.13 & 21.87 & 78.13 & 9.01 & 36.27 & 23.09 & 1.57 & 1.05 & 22.18 & 8.00 & 2.77 & 7.97 & 7.60 & 0.61 \\
\hline V80 & 3.68 & 193.89 & 47.44 & 3.04 & 17.03 & 82.97 & 6.42 & 36.11 & 19.23 & 1.88 & 1.27 & 21.70 & 9.10 & 2.39 & 5.15 & 4.07 & 0.60 \\
\hline V81 & 3.11 & 131.33 & 36.89 & 3.66 & 12.90 & 87.10 & 5.78 & 38.53 & 16.07 & 2.40 & 1.37 & 28.50 & 7.71 & 3.69 & 4.41 & 3.23 & 0.74 \\
\hline V82 & 2.93 & 124.78 & 50.44 & 4.03 & 32.77 & 67.23 & 11.26 & 40.94 & 20.28 & 2.02 & 1.39 & 27.93 & 6.33 & 4.41 & 9.87 & 7.11 & 0.68 \\
\hline V83 & 3.34 & 142.78 & 47.78 & 2.79 & 15.23 & 84.77 & 8.21 & 37.46 & 19.90 & 1.88 & 1.14 & 22.68 & 8.34 & 2.72 & 7.07 & 6.19 & 0.61 \\
\hline V84 & 4.08 & 126.11 & 48.00 & 2.82 & 16.97 & 83.03 & 7.36 & 37.45 & 17.40 & 2.15 & 1.01 & 25.20 & 7.04 & 3.58 & 6.35 & 6.28 & 0.67 \\
\hline V85 & 3.48 & 125.56 & 54.22 & 2.80 & 27.70 & 72.30 & 7.19 & 34.33 & 17.77 & 1.93 & 0.72 & 21.85 & 5.57 & 3.92 & 6.47 & 8.98 & 0.64 \\
\hline V86 & 2.63 & 160.44 & 53.11 & 2.61 & 18.97 & 81.03 & 8.40 & 35.84 & 19.82 & 1.81 & 1.09 & 23.57 & 8.09 & 2.91 & 7.31 & 6.72 & 0.66 \\
\hline V87 & 3.00 & 173.11 & 42.56 & 3.70 & 27.70 & 72.30 & 5.92 & 32.85 & 17.92 & 1.83 & 1.02 & 20.66 & 7.92 & 2.61 & 4.90 & 4.82 & 0.63 \\
\hline V88 & 3.30 & 133.00 & 54.78 & 2.83 & 22.60 & 77.40 & 7.07 & 32.70 & 21.49 & 1.52 & 1.37 & 26.08 & 7.60 & 3.43 & 5.70 & 4.15 & 0.80 \\
\hline V89 & 3.40 & 131.44 & 47.89 & 3.53 & 24.73 & 75.27 & 4.75 & 28.92 & 15.92 & 1.82 & 1.29 & 21.85 & 6.13 & 3.56 & 3.46 & 2.68 & 0.76 \\
\hline
\end{tabular}

Leaf length (Lo.P), pinna number (No.Pe), pinna length (Lo.Pe), pinna width (La.Pe), humidity (Hu), dry matter (Ms), fruit weight (P.F), fruit length (Lo.F), fruit width (La.F), length / width of fruit (Lo/La.F), seed weight (P.G), seed length (Lo.G), seed width (La.G), length / width of seed (Lo/La.G), weight of pulp (P.Pu), weight of pulp / weight of seed (P.Pu/P.G) and length of seed / length of fruit (Lo.G/Lo.F).

Longitud de la hoja (Lo.P), número de pinnas (No.Pe) longitud de la pinna (Lo.Pe), ancho de la pinna (La.Pe), humedad (Hu), materia seca(Ms), peso del fruto (P.F), longitud del fruto (Lo.F), ancho del fruto (La.F), longitud / ancho del fruto (Lo/La.F), peso de la semilla (P.G), longitud de la semilla (Lo.G), ancho de semilla (La.G), longitud / ancho de la semilla (Lo/La.G), peso de la pulpa (P.Pu), peso de la pulpa / peso de la semilla (P.Pu/P.G) y longitud de la semilla/ longitud del fruto (Lo.G/Lo.F). 


\section{RESULTS}

Mean values of morphometric characters studied are reported in Table III. Data show large variability between cultivars.

The overall differences in morphological variables between cultivars were examined by principal component analysis. The first component accounted for about $27 \%$ of the total variability, while the cumulative variance explained by the first two and the three components were about $44 \%$ and $55 \%$, respectively (Table III).

We found also their positions with respect to the fruits and the leaf (see Fig. 2), and especially of the quantities values studied, that 12 parameters (variables) have a good representation among the 17 parameters which deliberated.

This results was obtained by using the theory of the parameters square cosinus.

The abbreviations names are found in Fig. 3, and their square cosinus values were taken in two axis, the most important values are the sum of square cosinus of the first and second axis. it's very important to take the values of the sum square cosinus bigger than 0.70 as well repported,to have an excelent representation.

The values obtained were the following: pulp weight $(0,865)$, which is the highest value; fruit weight $(0,851)$, fruit length/fruit width $(0,754)$, fruit length $(0,746)$, weight of pulp seed $(0,675)$, the coefficient seed length/width $(0,576)$, dry matter $(0,516)$, humidity $(0,513)$, fruit width $(0,449)$, seed length $(0,426)$, seed width $(0,305)$, and the seed length/fruit length $(0,393)$.

The results show that there are only 18 varieties, and by using this theory of square cosinus of the variables, shows that:

Class Bezoul El Khadem (V16) has been taken the value of square cosine of the category of the varieties equal to 0.890, Haloua (V69) 0.785, Tanteboucht (V71) 0.771, Guelb Echa (V67) 0.764, Ghazi (V65) 0.755, Bedai (V13) 0.745, Tichtat (V37) 0.712, Rotebet Abdelah (V35) 0.690, Daglet Azzi (V25) 0.684, Guelb Echa (V68) 0.681, Rebib El Ghars (V51) 0.656, Sbaa Laroussa (V76) 0.655, Halouat Saada (V22) 0.652, Arechti (V7) 0.638, Deglet Nour (V28) 0.635, Baydh Lahmam (V41) 0.634, Baydh Ghoul (V12) 0.620 and Bouzerou (V21) the square cosinus of the category equal to 0.603 .

By observing the correlation circle in Figure 3, the traits have shown a convergence, between the variables which are: Humidity $(\mathrm{Hu})$ was in creasing relationship with weight of pulp (P.Pu), fruit weight (P.F), coefficients of the weight/ seed (P.Pu/P.G), fruit width (La.F), and with decreasing relationship by (Ms), as the first group.

The second group: fruit width (Lo.F), coefficient length of fruit/width (Lo/La.F), the coefficient seed length/width (Lo/La.G) and seed length (Lo.G), these above traits have a common effect with themselves, but it remains the representation of this group average compared to the first set of variables.

Axis 1 (F1): The traits that have a good representation are: the weight of pulp (0.763), fruit weight $(0.747)$, the coefficient weight of pulp/seed (0.631), the fruit length $(0.582)$, and to a lesser extent a proportion of humidity (0.390) was find, the proportion of dry matter (0.390), the coefficient the length of the seed/fruit length (0.391), and the coefficient the length of fruit/width (0.225).

Axis 2 (F2): The parameters which have the middle representation are the parameters length/width $(0.529)$, the coefficient length of seed/width (0.444), the fruit width (0.412), the seed length $(0.310)$ and the seed width $(0.252)$.

To verify the overlap of the traits, the correlation coefficient was studied, as described in Figure 3: results show the existence of liaisons and the counter liaisons between certain traits.

From the vegetative parameters: leaf length (Lo.P) increases with the pinnae number (No.Pe). In the traits fruiting: humidity $(\mathrm{Hu})$ has increased with the fruit weight, counter productive with the proportion of dry matter (Ms), finally it has an inverse relationship with the pulp weight (P.Pu). Fruit weight (P.F) has increasing relationship with each of the fruit length (Lo.F), the pulp weight (P.Pu), coefficient weight of flesh/seed (P.Pu/P.G), and counterproductive with the coefficient length of the seed/ length of the fruit (Lo.G/Lo.F). However the fruit length (Lo.F) has an increasing relationship with each of the coefficient length/width of fruit (Lo/La.F), the seed length (Lo.G), the pulp weight (P.Pu), but counterproductive with the coefficient of length of seed/length of the fruit (Lo.G/ Lo.F). On the other side, the fruit width has an inverse relationship with the coefficient of length/width of fruit (Lo/ La.F).

Beside of that the coefficient of length/width of fruit (Lo/La.F) has an inverse relationship with the coefficient of seed length/fruit length (Lo.G/Lo.F). The seed length (Lo.G) has a growing relationship with the coefficient of length/width of the seed (Lo/La.G). And (Lo/La.G) has an inverse relationship with seed width (La.F), pulp Weight (P.Pu) has increasing relationship with the coefficient pulp weight of the seed (P.Pu/P.G), and counterproductive with the coefficient of the seed length/fruit length (Lo.G/ Lo.F), and (Lo.G/Lo.F) it has an inverse relationship with coefficient pulp weight on the seed (P.Pu/P.G).

According to the kinship which has been described in Figures 4 and 5, there are 3 homogeneous groups, and this heterogeneity has been caused by the most important variables in the plan consisting of two axes (F1 and F2), and this division with respect to the Pearson coefficient $=0.982$, where each group will be composed of the following:

Group 1: the largest groups containing 48 varieties (components), and these varieties are similar with each other in this set of the variables: the seed width (La.G), fruit 
TABLE IV. Value of variations accumulated for the compounds F1, F2 and F3 resulting from the analysis.

TABLA IV. Valor de las variaciones acumuladas del F1, F2 y F3 compuestos resultantes del análisis.

\begin{tabular}{cccc}
\hline AXes & EigenValues & Variability (\%) & ACCuMULATED (\%) \\
\hline F1 & 4,580 & 26,941 & 26,941 \\
F2 & 2,892 & 17,012 & $\mathbf{4 3 , 9 5 3}$ \\
F3 & 2,000 & 11,762 & 55,715 \\
\hline
\end{tabular}

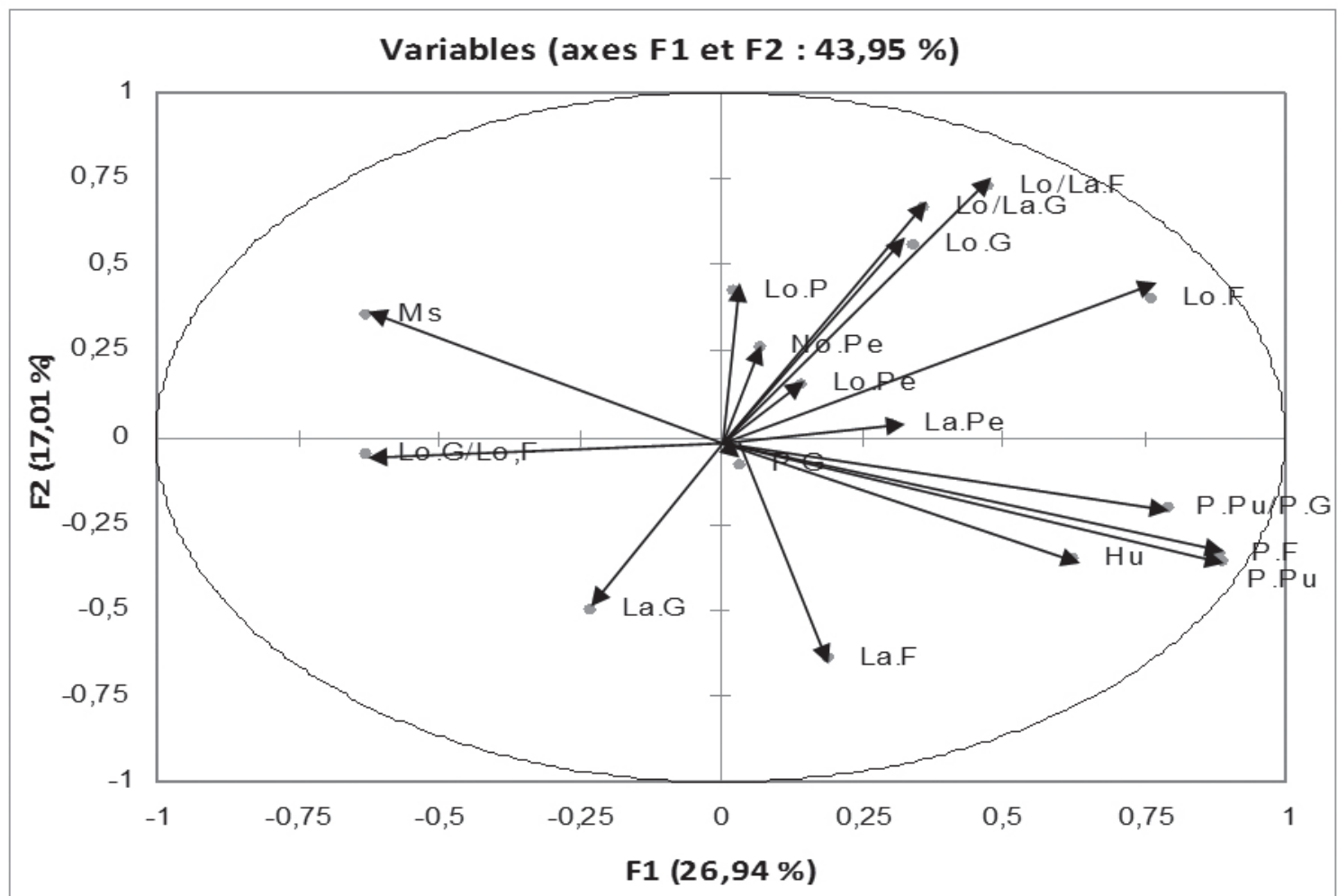

FIGURE 3. Correlation circle shows the relationship of the parameters and factorial axis

FigURA 3. Circulo de correlación mostrando la relación entre los parámetros y los ejes factoriales

width (La.F), humidity (Hu), coefficient of weight of flesh/ seed (P.Pu/P.G), the coefficient seed length/fruit length (Lo.G/Lo.F), fruit weight (P.F), and the pulp weight (P.Pu).

Group 2: composed by 34 varieties, which have the similarity in the following set of variables: the fruit length (Lo.F), the seed length (Lo.G), the proportion of dry matter (Ms), the coefficient of length/width of seed (Lo/La.G), and the coefficient length/width of fruit (Lo/La.F).

Group 3: Is composed of 3 varieties.

With regard to eigenvalue equal $43.95 \%$, this value will be considered acceptable made it possible to find $58 \%$ of the first group down the coordinate's axis, and $67 \%$ above the coordinate's axis.

\section{DISCUSSION}

The results obtained show differences between the varieties, especially fruiting characteristics, while vegetative traits are not representative of the varieties.

Overall analysis selected leaves characteristics shows a high diversity of cultivars. Certainly this phenotypic variability reflects a genetic diversity while the environment effect was eliminated. This diversity is due to genetic recombination during the sexual reproduction, given that each cultivar derives from a unique seed, cloned thereafter by vegetative multiplication (Elhoumaizi et al. 2002).

The morphological studies of date palm have always 
been considered difficult to undertake because they require a large set of phenotypic data and because they are varied due to the environment effect (Munier 1973). The present study has sifted morphological characters that are not controlled by edaphic or climatic factors. Several previously published data have indicated some morphological characters but the major has an adaptive response to the environment (Nixon 1950).

The geographic origin was not a determinant criterion for cultivars aggregation, because the names of cultivars change frequently from one oasis to the other because synonyms and homonyms may be encountered.

The majority of the phenotypic studies on date palm aimed at studying the spectrum of the genetic variation but they cannot allow definitive discrimination between cultivars, fruit quality and plant behavior. However, the study of Saaidi (1979) and Elhoumaizi et al. (2002) highlighted a strong morphological resemblance between some date palm cultivar known for their resistance to Bayoud disease.

Date palm selection by peasants is often based on fruit characteristics. Hence, this study highlighted the relationship between vegetative and fruit characteristics which may be genetically related. Vegetative tools are so important in genotype evolution process; they are decisive in date palm cultivar selection and adaptation (Hamza et al. 2009).

Previous studies have looked at biochemical and molecular markers of cultivars. The use of isozymes permit discrimination of some cultivars but these methods are not in routine use (Baaziz \& Saaidi 1998, Bennaceur et al. 1991). In addition, these methods gave contradicting results when applied to date-palm trees and other plants (Chevreau 1990, Elhoumaizi et al. 1993).

Research in molecular markers is taking the lead in this context (Aitchitt et al. 1995, Corniquel \& Mercier 1997, Lewis et al. 2000). However, these methods are at a preliminary stage with regard to date-palm research, and they have been tested on a limited number of cultivars (Benabdellah et al. 2000, Trifi et al. 2000). Despite the large number of cultivars evaluated by Sedra et al. (1998) (43 cultivars using RAPD markers), the study could not identify a significant discriminating criteria between different cultivars. In addition, this study overlooked some important cultivar like 'Mekt', 'Oumnhel', 'Racelahmar' and 'Mestali'. The currently existing biochemical and molecular data do not allow definitive discrimination between cultivars, fruit quality and resistance to Bayoud disease.

Morphological, isozyme and molecular data of date palm cultivars gave different results (Elhoumaizi et al. 1993, Bendiab et al. 1993, Sedra et al. 1998).

Vegetative characteristics can be considered as a principle to know the differences between the date palm varieties (Djerouni et al. 2015). Moreover, Mohamed Lemine et al. (2014) affirmed in their study of 28 Mauritanian date palm varieties that there are differences in the physical characteristics of the fruits. As Haider et al. (2015) concluded in their study on the sixteen Pakistani palm dates varieties from different origins in terms of vegetative characteristics that the pinna number, length and width and the Leaf palm length are characteristics that help to differentiate between the types of date palm. According to Saker et al. (2010), the anatomical study of date palm fruits is a scientific measure that helps to find the similarities between the female varieties. Hamadi et al. (2009) have confirmed in their study of some Tunisian date palm varieties that the vegetative characteristics like thorns length, the length of thorns area and the leaf palm length are appropriate characteristics that show the similarities and the differences between the female palms. Since the vegetative characteristics are only the expression of genes, the molecular analysis proved the phenotypic differences between palm date varieties (Ahmed et al. 2006, Zhao et al. 2013).

The same situation was observed in other plants, because a short distance calculated with RFLP markers always corresponds to a short morphological distance; however, a long distance calculated with the molecular markers can correspond to a long or a short morphological distance. This relation could be explained with the fact that a morphological character can be resulted of various combinations of genes (Grivet \& Noyer 1999). The morphological study is indispensable as far as programs of selection and improvement of date palm are concerned.

This method of taxonomy cannot resolve definitively the cultivar identification problem. In fact, many different populations may have the same aspect in spite of their different genotypes.

Future studies should consider the possible relations of other important phenotypic markers related to the tolerance towards oases stress. This should be backed up by others studies such as molecular ones to provide reliable tools for measuring genetic divergence.

\section{ACKNOWLEDGEMENTS}

The authors are thankful to the Director of ITDAS-Biskra for providing their facilities. Thanks must be given to all staff of INRA-Biskra experimental station.

\section{REFERENCES}

Ahmed, M.M., S.S. Soliman \& E.H. Elsayed. 2006. Molecular Identification of Some Egyptian Date Palm Males by females varieties (Phoenix dactylifera L.) Using DNA Markers. Journal of Applied Sciences Research 2(5): 270275.

Aitchitt, M., S.H. Mantell, M. Thangavelu \& C.C. Ainworth. 1995. Cloning date-palm (Phoenix dactylifera L.) DNA and characterisation of low, medium and high DNA 
sequences. Elaeis 7: 57-63.

Anonyme. 2003. La Monographie de la wilaya de Biskra. Direction d'aménagement de territoire et de planification. $7 \mathrm{pp}$.

Anonyme. 2005. Descripteurs du palmier dattier (Phoenix dactylifera L.). International Plant, Genetic Resources Institute (I.P.G.R.I.), Rome, Italie. 71 pp.

AnONYme. 2011. La production des dattes dans en Algérie. Ed. FAO, Organisation des Nations Unies pour l'Alimentation et l'Agriculture. $41 \mathrm{pp}$.

BAAziz, M. \& M. SAAIDI. 1998. Preliminary identification of datepalm cultivars by esterase isoenzymes and peroxidases activities. Canadian Journal of Botany 66: 89-93.

Barrett, H.C. 1975. Evaluation of date Cultivars for dessert. Fruit Varieties Journal 29(3): 55-58.

Bashah, M.A. 1996. Date Varieties in the Kingdom of Saudi Arabia. Guidance booklet: palms and dates. King Abdulaziz University Press, Riyadh, Saudi Arabia. 1225$1319 \mathrm{pp}$.

Belguedj, M. 2002. Caractéristiques des cultivars de dattier dans les palmeraies du Sud-Est Algérien, INRA, Algérie. 211 pp.

Belguedj, M. \& A. Tirichine. 2011. Caractéristiques des cultivars de Ghardaia Algérien, INRA, Algérie. 175 pp.

Belguedj, M., A. Tirichine \& M. Guerradi. 2008. La culture du palmier dattier dans les oasis du Ghardaia (Algérie). Alger: INRA, Algérie. 21 pp.

Benabdellah, A., K. Stiti, P. Lepoivre \& P. Du Jardin. 2000. Identification de palmier dattier (Phoenix dactylifera L.) par l'amplification aléatoire d'ADN (RAPD). Cahiers Agricultures 9: 103-107.

Bendiab, K., M. BaAziz, Z. Brakez \& M.H. Sedra. 1993. Correlagertion of isoenzyme polymorphism and Bayoud disease resistance in date-palm cultivars and progeny. Euphytica 65: 23-32.

Bennaceur, M., C. Lanaud, M.H. Chevalier \& N. Bounaga. 1991. Genetic diversity of the date-palm (Phoenix dactylifera L.) from Algeria revealed by enzyme markers. Plant Breeding 107: 56-69.

BRown, T.W. 1924. Date palm in Egypt. Technology and Science. Services, Horticulture. Section, Bulletin 43. Ministry of Agriculture, Cairo.

Chevreau, E. 1990. Biotechnologies et amélioration du pommier et du poirier. Arboriculture fruitière 429: 19-24.

Corniquel, B. \& L. Mercier. 1997. Identification of date-palm (Phoenix dactylifera L.) cultivars by RFLP: partial characterization of a DNA probe that contains a sequence encoding a zinc finger motif. International Journal of Plant Sciences 158: 152-156.

Djerouni, A., A. Chala, A. Simozrag, R. Benmhaia \& M. Baka. 2015. Evaluation of male palms used in pollination and the extent of its relationship with cultivars of date-palms (Phoenix dactylifera L.) grown in region of Oued Righ, Algeria. Pakistan Journal of Botany. 47(6): 2295-2300.

Dowson, V.H.W. 1923. Dates and Date Cultivation of Iraq, Part III, The varieties of date palms in the Shalt Al Arab Agriculture Directorate of Mesopotamia, Memoir III, Heffer, Cambridge. 294 pp.

Elhoumaizi, M.A., M. SAaidi \& M. BaAziz. 1993. Morphometric and isoenzymatic study of six date-palm cultivars cultivated in Marrakech and Zagora. Alawamia 82: 151163.

Elhoumaizi M.A., M .SaAidi, A. Oihabi \& C.cilas. 2002. Phenotypic diversity of date palm cultivars (Phoenix dactylifera L.) from Morocco. Genetic Resources and Crop Evolution 49: 483-490.

Grivet, L. \& J.L. Noyer. 1999. Les méthodes de marquage biochimique et moléculaire. In: P. Hamon, M. Segun, X. Perrier \& J.C. Glasmann (eds.), Diversité Génétique Des Plantes Tropicales Cultivées. pp. 13-41. CIRAD Press.

Haider, M.S., I.A. Khan, M.J. Jaskani, S.A. Naqvi, M. Hameed, M. Azam, A.A. Khan \& J.C. Pintaud. 2015. Assessment of morphological attributes of date palm accessions of diverse agro-ecological origin. Pakistan Journal of Botany 47(3): 1143-1151.

Hammadi, H., R. Mokhtar, E. Mokhtar \& F. Ali. 2009. New approach for the morphological identification of date palm (Phoenix dactylifera L.) cultivars from Tunisia. Pakistan Journal of Botany 41(6): 2671-2681

Hannachi, S., D. Khitri, A. Benkhalifa \& R.A.B. De La Perriere. 1998. Inventaire variétal de la palmeraie algérienne, Ed. ANEP, Algérie, $155 \mathrm{pp}$.

Johnston, R.J. 1978. Multivariate Statistical Analysis in Geography: A Primer on the General Linear Model. Longman, London, UK. 122 pp.

Khadhi, M.S., M. Ben Salah, \& H. Jeder. 1995. Les composantes de l'agriculture oasienne. In: G. Coudé-Gaussen \& P. Rognon (éds.), Désertification et aménagement au Maghreb. Paris, L'Harmattan. 193 pp.

Lewis, C.E., W. BAKer \& C.B. Assumussen. 2000. DNA and palm evolution. Palms 44: 19-24.

MaInLEY, B.F.J. 1994. Multivariate statistical methods: a primer. Chapman \& Hall, Bury, St. Edmonds, Suffolk, 215 pp

Mohamed Lemine, F.M., A. Samb, Z.O. Bouna, M. Vall, O. Mohamed Ahmed, T.O. Dueh \& A.O.M.O. Boukhary. 2014. African Journal of Agricultural Research 9(28): 2167-2176.

Munier, P. 1973. Le palmier dattier. Techniques agricoles et productions tropicales. Maisonoeuvre et Larose edition, Paris. 221 pp.

Nixon, R.W. 1950. Imported cultivars of dates in the United States U.S. Department of Agriculture Circular no. 834, U.S. Department of Agriculture, Washington, 144 pp.

Rhouma, A. 1994. Le palmier dattier en Tunisie 1: le patrimoine genetique. Vol.1 Arabasque, Tunis. 294 pp.

SAAIDI, M., 1979. Contribution a la lutte contre le Bayoud, fusariose vasculaire du palmier dattier. Thèse de Doctorat, Université de Dijon. France. 140 pp.

SaKr, M.M., I.M. Abu ZeID, A.E. Hassan, A.G.I.O. BaZ \& W.M. Hassan. 2010. Identification of some Date Palm (Phoenix dactylifera L.) cultivares by fruit characters. Indian Journal of Science And Technology 3(3): 338-343.

Sedra, M.H., P. Lashermes, P. Trouslot, M.C. Combes \& S. Hamon. 1998. Identification and genetic diversity analysis of date-palm (Phoenix dactylifera L.) varieties from Morocco using RAPD markers. Euphytica 103: 7582.

Trifi, M., A. Rhouma \& M. Marrakchi. 2000. Phylogenetic relationships in Tunisian date-palms (Phoenix dactylifera L.) germoplasm collection using DNA amplification 
fingerprinting Agronomie 20: 665-671.

ZAID, A. \& P.F. Wet. 1999. Botanical and Systematic description of the date palm. In: A. Zaid (ed.), Date Palm Cultivation. pp. 1-28. FAO, Rome.
Zhao, Y., R. Williams, C.S. Prakash \& H. Guohao. 2013. Identification and characterization of gene-based SSR markers in date palm (Phoenix dactylifera L.). BMC Plant Biology 1-8.

Appendix A. Names and abbreviations of cultivars of Phoenix dactylifera used in this study.

APÉNDICE A. Los nombres y las abreviaturas de los cultivares Phoenix dactylifera usados en este estudio.

\begin{tabular}{|c|c|c|c|c|c|}
\hline Code & Cultivar & CODE & Cultivar & CodE & Cultivar \\
\hline V1 & Abdelazaz & V31 & Derjini & V61 & Fliachia \\
\hline $\mathrm{V} 2$ & Ain El Fés & V32 & D'For El Gat & V62 & Garn Ghazel \\
\hline V3 & Saout Bghal & V33 & D'Guel Arechti & V63 & Lokzi \\
\hline V4 & Alig & V34 & Adjina & V64 & Ghars \\
\hline V5 & Amari & V35 & Rotebet Abdelah & V65 & Ghazi \\
\hline V6 & Arar & V36 & D’Guel Boujadi & V66 & Tati Bent Nouh \\
\hline V7 & Arechti & V37 & Tichtat & V67 & Kantichi \\
\hline V8 & Khnafre & V38 & D'Guel Bouzouaid & V68 & Guelb Echa \\
\hline V9 & D'Guel Eljayh & V39 & D'Guel Daim & V69 & Haloua \\
\hline V10 & Assala & V40 & D’Guel Debdab & V70 & Halouat Loulache \\
\hline V11 & Baar El Djeaach & V41 & Baydh Lahmam & V71 & Tanteboucht \\
\hline V12 & Baydh Ghoul & V42 & D'Guel El Bar & V72 & Noyet Rass El Thaour \\
\hline V13 & Bedai & V43 & Takarmoust & V73 & Tafezuine \\
\hline V14 & Bent Merague & V44 & D'Guel Hamlaoui & V74 & Hamrayet El Ghareb \\
\hline V15 & Besbassi & V45 & Khaira D'Guel & V75 & Noyet Deglet Nour \\
\hline V16 & Bezoul El Khadem & V46 & Zogar Mogar & V76 & Sbaa Laroussa \\
\hline V17 & Bouarous & V47 & Zemachi & V77 & Horra \\
\hline V18 & Ech Chouaib & V48 & D'Guel Maroufi & V78 & Mech Degla \\
\hline V19 & Boulantate & V49 & Rass El Thaour & V79 & Itima \\
\hline V20 & Bouzenzen & V50 & Mezith & V80 & Jaouzia \\
\hline $\mathrm{V} 21$ & Bouzerou & V51 & Rebib El Ghars & V81 & Sokria \\
\hline V22 & Halouat Saada & V52 & Dalgla touila & V82 & Sokriet Hassanine \\
\hline V23 & Dahbia & V53 & Tinicine & V83 & Khdraye \\
\hline V24 & Degla Baidha & V54 & D'Guel Souareg & V84 & Safraye \\
\hline $\mathrm{V} 25$ & Daglet Azzi & V55 & Oudane & V85 & Rotbet Cheikh Amar \\
\hline V26 & Khdraya & V56 & D'Guel Trik & V86 & Khoudri \\
\hline V27 & Daglet Med Tahar & V57 & Timdjouhart & V87 & Kseba \\
\hline V28 & Deglet Nour & V58 & Thouri & V88 & Laoun Litima \\
\hline V29 & Daglet Ziane & V59 & Ech El Oued & V89 & Menakher \\
\hline V30 & Daldala & V60 & Tebet Nouh & & \\
\hline
\end{tabular}

Recibido: 27.07 .15

Aceptado: 10.03 .16 